\title{
A call for individualized evacuation strategy for floods: a case report of secondary surgical site infection in a post-surgery breast cancer patient in Fukushima, Japan, following Typhoon Hagibis in 2019
}

\author{
Akihiko Ozaki ${ }^{1}$, Yoshiaki Kanemoto ${ }^{1}$, Masahiro Wada ${ }^{1}$, Tomohiro Kurokawa ${ }^{1}$, Ayumu \\ Kawamoto $^{2}$, Toyoaki Sawano ${ }^{1}$, Divya Bhandari ${ }^{3}$, Masaharu Tsubokura ${ }^{4}$, Tetsuya \\ Tanimoto $^{1}$, Tomozo Ejiri ${ }^{1}$, and Norio Kanzaki ${ }^{1}$ \\ ${ }^{1}$ Jyoban Hospital of Tokiwa Foundation \\ ${ }^{2}$ University of Szeged Faculty of Medicine \\ ${ }^{3}$ The University of Tokyo Graduate School of Medicine Faculty of Medicine \\ ${ }^{4}$ Fukushima Medical University School of Medicine
}

October 27, 2020

\begin{abstract}
Cancer patients, particularly those post-surgery, are prone to skin infection following immersion in flood waters. We reported secondary surgical site infection in a postoperative 80-year-old post-operation breast cancer patient affected by Typhoon Hagibis. Individualized responses based on individual and environmental risks are crucial to alleviate the damage of disasters.
\end{abstract}

\section{Clinical Case Reports, Case Report}

\section{Title:}

A call for individualized evacuation strategy for floods: a case report of secondary surgical site infection in a post-surgery breast cancer patient in Fukushima, Japan, following Typhoon Hagibis in 2019

\section{Authors:}

Akihiko Ozaki ${ }^{1}$, Yoshiaki Kanemoto ${ }^{2}$, Masahiro Wada ${ }^{1,3}$, Tomohiro Kurokawa ${ }^{2}$, Ayumu Kawamoto ${ }^{4}$, Toyoaki Sawano ${ }^{5,6}$, Divya Bhandari ${ }^{7}$, Masaharu Tsubokura ${ }^{6}$, Tetsuya Tanimoto ${ }^{8}$, Tomozo Ejiri ${ }^{2}$, Norio Kanzaki $^{2}$

\section{Affiliations:}

${ }^{1}$ Department of Breast Surgery, Jyoban Hospital of Tokiwa Foundation, Iwaki, Fukushima, Japan

${ }^{2}$ Department of Surgery, Jyoban Hospital of Tokiwa Foundation, Iwaki, Fukushima, Japan

${ }^{3}$ Department of Breast Surgery, Sano Kosei General Hospital, Sano, Tochigi, Japan

${ }^{4}$ Faculty of Medicine, University of Szeged, Szeged, Hungary

${ }^{5}$ Department of Surgery, Sendai City Medical Center, Sendai Open Hospital, Sendai, Miyagi, Japan.

${ }^{6}$ Department of Radiation Health Management, Fukushima Medical University School of Medicine, Iwaki, Fukushima, Japan

${ }^{7}$ Community and Global Health, Graduate School of Medicine, the University of Tokyo, Tokyo, Japan 
${ }^{8}$ Department of Internal Medicine, Jyoban Hospital of Tokiwa Foundation, Iwaki, Fukushima, Japan

\title{
Corresponding author:
}

Akihiko Ozaki, MD, PhD

Department of Breast Surgery, Jyoban Hospital of Tokiwa Foundation, 57 Kaminodai, Jyoban-KamiyunagaYamachi, Iwaki, Fukushima, Japan.

Email address: aozaki-tky@umin.ac.jp.

Telephone: 81-244-22-3181

Fax: 81-244-22-8853

Funding: None available

\begin{abstract}
:
Cancer patients, particularly those post-surgery, are prone to skin infection following immersion in flood waters. We reported secondary surgical site infection in a postoperative 80-year-old post-operation breast cancer patient affected by Typhoon Hagibis. Individualized responses based on individual and environmental risks are crucial to alleviate the damage of disasters.
\end{abstract}

\section{Key words}

Breast Neoplasms; Japan; Climate Change; Cyclonic Storms; Floods

\section{Key Clinical Message}

Recognition of Individual and environmental risks is crucial to alleviate the damage of disasters. In particular, an awareness of the flood risks in patients' residences is important for patients and their healthcare professionals.

\section{Introduction}

Flood frequency has been increasing globally due to climate change. ${ }^{1}$ When flood water submerges residential districts, it is affected by sewage and can easily cause skin infections in the affected population. ${ }^{2}$ When considering populations vulnerable to skin infections following flooding, the risk may be higher in post-surgery cancer patients than the general population because the immune systems of cancer patients are suppressed ${ }^{3}$ and they are particularly compromised after chemotherapy, radiotherapy, or surgery. ${ }^{2}$ Incisional wounds are especially susceptible to surgical site infections. However, little information is available concerning measures to prevent such infections among these populations following floods.

Typhoon Hagibis, which occurred on October 6, 2019 and landed on Japan's main island on October 12, 2029, killed approximately one hundred citizens and caused various other injuries. ${ }^{4}$ These damages occurred although typhoon warnings had been repeatedly disseminated in various media outlets beforehand. This typhoon was a Category 5 (the highest according to the Saffir-Simpson Hurricane Wind Scale) and was one of few severe disasters that required government support in its recovery. Given this context, we present a post-operative breast cancer patient who was affected by flood water caused by Typhoon Hagibis in order to plan specific measures to ensure the safety of cancer patients from flood damage in the future.

\section{Case description}

An 80-year old patient living with her son-in-law and grandson in Iwaki City, Fukushima, with chronic renal diseases of unknown cause, found a lump on the top of her right nipple and initially visited her primary physician in March 2019. She was then referred to our breast surgery outpatient office on June 17, 2019 after several consultation cancellations. An in-depth diagnostic workup confirmed cancer of the right breast with anatomical clinical stage of cT2N0M0 StageIIA. We carried out mastectomy, sentinel lymph node biopsy with a positive lymph node metastasis, and subsequent axillary resection on September 25, 2019. The 
postoperative condition was stable, and she was discharged on October 11, 2019, which was the day before Typhoon Hagibis hit Iwaki City (post-operative day 16). Although she was advised to stay in the hospital until the typhoon passed by the city, she insisted on discharge that day.

At midnight on October 12, 2019, the typhoon heavily hit the city and destroyed 5,927 residences and 3,111 non-residential buildings, submerged 5,721 buildings, and killed 12 people. Her single-story residence was located 13 meters above sea level and only 100 meters away from the embarkments running along the Natsui River. The flood hazard map that estimates areas that would be submerged by floods with a scale of once in one thousand years is open access on the municipality website and clearly showed that her residence would become submerged up to 5 to 10 meters high due to overflow of the nearby Natui River. This occurred because the typhoon destroyed several of the river's embarkments at 12:30am on October 13, 2019. While Iwaki City issued an evacuation order for those living around the river at 9:40pm on October 12, 2019, she reportedly did not evacuate from her residence because she was not aware of that warning. The flood water submerged her residence soon after the destruction of embarkments when she was asleep. The water level rapidly reached approximately two meters high, and she managed to survive by climbing on furniture her son-in-law piled up for her. Despite her efforts to cover her wound, it was immersed by contaminated flood water. The water smelled rotten, probably because of sewage that overflowed from the sewers, given that scattered manhole covers were observed around her residence the next day.

On October 13, 2019, she complained of appetite loss and visited our emergency department the next day (October 14, 2019). On physical examination, she was afebrile and her surgical site was not apparently infected, but she was hospitalized due to appetite loss and destruction of her residence. After admission, her surgical site gradually became red, swollen, and edematous, highly suggestive of secondary infection. On October 16, we inserted a drainage tube into the surgical wound and started administration of cefazolin $1 \mathrm{~g}$ three times per day. We failed to take a culture of the fluid sample. Further discussion revealed that she had not carefully read the flood hazard map before the typhoon. Her condition gradually improved and she was discharged on November 5, 2019. After discharge, she abandoned her original residence because of persistent smells and moved to a nearby apartment building following several relocations.

\section{Discussion}

This case describes a skin infection in a postoperative breast cancer patient due to an immersion of surgical site with contaminated flood water following Typhoon Hagibis. Her discharge took place on the day before the typhoon hit Iwaki City, and she had characteristics that would make her vulnerable to floods, such as advanced age, breast cancer, and post-operative status. In this regard, we believe that there might have been some room for interventions to prevent her from incurring such severe flood damage.

Of note, a flood hazard map covering areas that would be submerged in floods was open access and widely available in Iwaki City. In Japan, local municipalities usually publish flood hazard maps on its website and on paper for community distribution. Further, the Geospatial Information Authority in Japan, similar to the Federal Emergency Management Agency in the United States and Ministry of Environment in France, prepares a website called "Kasaneru (Superimposing) Hazard Map [in Japanese]" where people can freely evaluate risks of flooding and landslides in basically any location in Japan. According to a municipality-based hazard map, her neighborhood was designated as a flood-prone area, while the hospital was not. Following Typhoon Hagibis, the patient's neighbors were all affected by flooding, but the hospital only incurred minor ceiling water leakage.

If we healthcare professionals had understood the information from the flood hazard map beforehand, we could have given her more appropriate advice when she wished to be discharged the day before the typhoon hit the city. Specifically, we could have more strongly persuaded her to stay at the hospital to prevent potential harm. Further, even when she declined our suggestion, we could have alternatively advised her to evacuate to residences of family members or friends living outside the flooding areas. Needless to say, some medical institutions exist in flood-prone areas, and this approach cannot always be generalizable.

For all residents including cancer patients, as is a lesson from this case, it is important to know whether their 
residences are located in flood-prone areas in ordinary times, but this may not be enough to guarantee a safe evacuation in situations when flood damage is anticipated. People are easily affected by normalcy bias, which is a tendency to consider that they themselves will not be affected by disasters or crises. ${ }^{5,6}$ In this regard, it is unclear whether people can successfully evacuate just because they were aware of the warning in a timely fashion. Rather, they may have been affected by normalcy bias, and somehow considered that they would not be affected by the flood. It is not always easy to overcome normalcy bias, but informal and formal support and ordinary-time preparedness would help people avoid this inherent mental tendency. ${ }^{6}$

Every natural disaster including and beyond flood is unique. In this respect, lessons gained from one disaster are not directly applicable to another. For instance, dozens of people died in floodwater in Kumamoto, Japan in July 2020. The damage was not caused by typhoons, but by a seasonal rain front, and it was obviously more difficult to predict the occurrence, timing, and potential locations than those of typhoons. Nonetheless, our ongoing mission is a continuous accumulation of evidence of any kind of health issue related to flooding to establish effective countermeasures to safeguard the health and well-being of cancer patients and other citizens.

In conclusion, we reported a case of secondary surgical site infection in a postoperative breast cancer patient affected by Typhoon Hagibis. Individualized responses based on individual and environmental risks are crucial to alleviate damage from disasters. In particular, an awareness of flood risks in patients' residences are important for patients and their healthcare professionals.

Conflict of interest : Akihiko Ozaki receives personal fees from MNES Inc, outside the submitted work. Also, Tetuya Tanimoto receives personal fees from MNES Inc, and Bionics co., ltd. outside the submitted work.

Ethical approval : Written informed consent was obtained from the patient for publication of this case report and any accompanying images.

Funding information: None

Acknowledgements: None

Author Contribution: AO wrote the manuscript. All authors conceptualized and designed the case report, and revised the paper. All authors read and approved the final manuscript.

\section{References}

1. Hirabayashi Y, Mahendran R, Koirala S, et al. Global flood risk under climate change. Nature Climate Change 2013;3:816-21.

2. Alderman K, Turner LR, Tong S. Floods and human health: a systematic review. Environ Int 2012;47:3747.

3. Whiteside TL. Immune suppression in cancer: effects on immune cells, mechanisms and future therapeutic intervention. Semin Cancer Biol 2006;16:3-15.

4. Ozaki A, Kanemoto Y, Morita T, et al. Nail Wound and Cellulitis Following Typhoon Hagibis in Fukushima, Japan. Disaster Med Public Health Prep 2020:1-3.

5. Ozaki A, Leppold C, Sawano T, et al. Social isolation and cancer management - advanced rectal cancer with patient delay following the 2011 triple disaster in Fukushima, Japan: a case report. J Med Case Rep 2017;11:138.

6. Ozaki A, Leppold C, Tsubokura M, et al. Social isolation and cancer management after the 2011 triple disaster in Fukushima, Japan: A case report of breast cancer with patient and provider delay. Medicine (Baltimore) 2016;95:e4027. 